\title{
A Case Study of Malignant Edema in Postpartum Mediterranean Buffaloes (Bubalus bubalis)
}

\author{
L. D'Angelo ${ }^{1 *}$, A. Martucciello ${ }^{1}$, C. Marianelli ${ }^{2}$, C. Grassi ${ }^{1}$, L. Schiavo ${ }^{1}$, F. Armas ${ }^{2}$, \\ D. Alfano ${ }^{1}$, D. Vecchio ${ }^{1}$ and E. De Carlo ${ }^{1}$
}

${ }^{1}$ Istituto Zooprofilattico Sperimentale del Mezzogiorno, National Reference Centre for Hygiene and Technologies of Water Buffalo Farming and Productions, Via Delle Calabrie 27, Salerno, Italy

${ }^{2}$ Unit of Emerging Zoonoses, Department of Food Safety, Nutrition and Veterinary Public Health, Istituto Superiore di Sanità, Viale Regina Elena 299, Rome, Italy

\begin{abstract}
The paper describes for the first time four cases of postparturient malignant edema in water buffaloes due to Clostridium septicum ( $\mathrm{Cl}$. septicum). The study was carried out on four primiparous buffaloes that showed swelling of perineal and perivulvar areas, fever and agalactia a few hours after calving. Two of them died within 20 hours after calving. The other two developed edema in the skeletal muscles of one leg and were treated with sulfadiazinetrimethoprim for 10 days. The clinical signs completely resolved. Culture and molecular investigations identified the pathogen isolated from exudate taken from the vulva as $\mathrm{Cl}$. septicum. The isolate showed multi-drug resistance. In the management of infection due to $\mathrm{Cl}$. septicum, timely diagnosis and the chirurgic curettage, associated with a broadspectrum antibiotic therapy, were found to be sufficient for the survival and recovery of the infected animals.
\end{abstract}

Keywords: Antibiogram, buffaloes (Bubalus bubalis), Clostridium septicum, malignant edema.

\section{INTRODUCTION}

Clostridia, which are Gram-positive, anaerobic, rodlike bacteria, are the cause of disease and death in humans and animals in most parts of the world. Clostridium septicum penetrates through lesions of the skin and mucosa and develops in an anaerobic environment, causing myonecrosis (malignant edema, gangrene), which may be rapidly fatal. This microorganism has been reported to be the cause of malignant edema in cattle [1,2], sheep, pigs and other species [3].

In ruminants, the most likely access route of $\mathrm{Cl}$. septicum is through breaches in the integrity of the mucosa of the vulva, vagina, and uterus, which may occur during calving. Contamination may take place directly from the environment or feces. Subsequently, the damaged mucosa may allow $\mathrm{Cl}$. septicum to penetrate to the internal tissues, while the bruising of the deep tissues creates the anaerobic conditions that favor its proliferation. Cl. septicum is a swarming bacterium, a feature that enables it to spread rapidly along the fascial planes of the muscles, thus causing progressive damage to the surrounding muscular and connective tissues, impaired movement and general discomfort. The course of the disease is acute/ peracute, with evident clinical signs appearing

*Address correspondence to this author at the Istituto Zooprofilattico Sperimentale del Mezzogiorno, National Reference Centre for Hygiene and Technologies of Water Buffalo Farming and Productions, Via Delle Calabrie 27, Salerno, Italy; Tel: 0039 0899257122; Fax: 0039089302699 ;

E-mail: luisa.dangelo@izsmportici.it within 24 hours and death ensuing 1-5 days after the onset. $\mathrm{Cl}$. septicum produces an alpha-toxin, the main toxin of this organism, which is necrotizing and hemolytic, causing increased capillary permeability. The primary structure of alpha-toxin shows $\sim 72 \%$ similarity with that of aerolysin (produced by Aeromonas hydrophila) and its mode of action is also similar [4]. The alpha-toxin causes rapid myonecrosis, interstitial hemorrhage, and edema when injected into mice [5] and microvascular collapse [6]. In addition to the lethal alpha-toxin, $\mathrm{Cl}$. septicum also produces a beta toxin (a ribonuclease with a cytolytic activity), a gamma toxin and a delta toxin (which exert hyaluronidase and hemolytic activities, respectively), a neuraminidase, hemagglutinin, a chitinase, a weak lipase and a sialidase [4]; although not lethal, these substances are thought to contribute to the pathogenesis of $\mathrm{Cl}$. Septicum-induced disease by facilitating tissue invasion and myonecrosis, and by reducing microvascular blood flow.

We describe for the first time, to the best of our knowledge, four cases of post-parturient malignant edema caused by $\mathrm{Cl}$. septicum in buffaloes (Bubalus bubalis).

\section{MATERIAL AND METHODS}

\section{Clinical Evaluation}

Four buffaloes from a herd of 1000 animals in the province of Salerno, southern Italy were primiparous and calved in January 2013. During clinical 
examination conducted a few hours after calving, they showed fever and agalactia. The vulva was massively swollen, taut, hot, crackling and painful on palpation, suggesting the presence of gas. Within 24 hours following calving, the edema spread to the skeletal muscles surrounding subcutaneous tissues, and to the muscles of one leg, causing the animals to drag the affected leg. Two of the four animals died within 20 hours of calving. The two surviving animals were treated 24 hours postpartum through lavage and curettage of the affected area with hydrogen peroxide mixed with betadine. An intramuscular injection of $10 \%$ ketoprofen ( $3 \mathrm{mg} / \mathrm{Kg} /$ day) for 3 days and sulfadiazinetrimethoprim $1 \mathrm{ml} / 10 \mathrm{~kg} /$ day) for 10 days were also administered. After treatment, the general health conditions of both animals improved. and necrosis of the superficial tissues in the area affected by the edema, with abundant tissue loss followed by healing by second intention. The area affected by necrosis was $30 \mathrm{~cm}^{2}$.

\section{Clostridium Identification by Polymerase Chain Reaction (PCR)}

Before treatment, samples of exudate were taken from each animal through sterile swabs, and tissue samples were taken from the edematous area of the vulva and thigh. The refrigerated samples were taken in 6 hours to the laboratory, where they underwent bacteriological and molecular examination. The samples were inoculated on $5 \%$ sheep blood agar plates and MacConkey agar. Incubations were performed at $37^{\circ} \mathrm{C}$ for $48-72 \mathrm{~h}$ in aerobic and anaerobic conditions on the blood agar plates and aerobically on MacConkey agar. The colonies observed after incubation in anaerobic conditions were swarming, spreading and hemolytic. Gram staining of these colonies revealed Gram-positive organisms of characteristic rod shape appearance. The isolates suggestive of the genus Clostridium [7] were identified through biochemical techniques (Vitek 2 Biomereux) and biomolecular investigation. Selected colonies were subjected to DNA extraction through the QIAamp DNA Mini Kit (Qiagen), followed by molecular analysis. Two primers (Forward 5'-CGGCTGGATCACCTCCTTTC-3' and Reverse 5'-ATCACGTCCTTCATCGGCTC-3') were designed by the Primer-BLAST utility (http://www.ncbi.nlm.nih.gov/tools/primer-blast/) to amplify a 460 base pair (bp) PCR product from the conserved sequences of the 16S-23S rDNA spacer regions of $\mathrm{Cl}$. septicum (accession no. AB040716). 0.2 $\mu \mathrm{M}$ of each primer was added to the PCR reaction. Amplification was performed in a total volume of $25 \mu \mathrm{L}$ by using GoTaq Green Master Mix (Promega Corporation, Madison, WI) following the manufacturer's instructions. The amplification profile was as follows: 2 min at $94^{\circ} \mathrm{C}$, followed by 30 cycles of $30 \mathrm{~s}$ at $94^{\circ} \mathrm{C}, 30$ $\mathrm{s}$ at $58{ }^{\circ} \mathrm{C}$, and $30 \mathrm{~s}$ at $72{ }^{\circ} \mathrm{C}$. After amplification, all reaction mixtures were analyzed through electrophoresis in a $2 \%$ agarose gel, stained with Gel Red Nucleic Acid Stain (Biotium Inc., Hayward, CA) and photographed. Since no Clostridium reference strains were available as $\mathrm{PCR}$ positive controls, the amplicon was purified by Montage PCR Centrifugal Filter Devices (Millipore, Billerica, MA) and directly sequenced by the ABI PRISM 310 Genetic Analyzer equipment using the BigDye Terminator v1.1 Cycle Sequencing Kit (Applied Biosystems, Foster City, CA). PCR primers were used for sequencing. The nucleotide sequence was then compared to the database by the BLAST program (http://www.ncbi.nlm.nih.gov/BLAST/). The DNA was confirmed as $\mathrm{Cl}$. septicum. No other clostridia were isolated, nor were other bacteria with pathogenic capability.

\section{Susceptibility Test}

After biomolecular confirmation, the susceptibility of the test isolates was evaluated by disk diffusion in agreement with Bauer [8] using commercially manufactured disks (Oxoid) available in the laboratory and containing ampicillin $(10 \mu \mathrm{g})$, penicillin (10IU), cephalexin $(30 \mu \mathrm{g})$, erythromycin $(15 \mu \mathrm{g})$, marbofloxacin $(5 \mu \mathrm{g})$, rifampin $(30 \mu \mathrm{g})$, amoxicillin $(25 \mu \mathrm{g})$, nalidixic acid $(30 \mu \mathrm{g})$, kanamycin $(30 \mu \mathrm{g})$, lincomycin $(2 \mu \mathrm{g})$, norfloxacin $(10 \mu \mathrm{g})$, spiramycin $(100 \mu \mathrm{g})$, colistin $(50 \mu \mathrm{g})$, gentamycin $(10 \mu \mathrm{g})$, tetracycline $(30 \mu \mathrm{g})$, sulfamethoxazole-trimethoprim $(25 \mu \mathrm{g})$. The results were recorded as 'sensitive' or 'resistant', in agreement with the Performance Standards for Antimicrobial Disk Susceptibility Tests, Clinical and Laboratory Standard Institute guidelines. Cl. septicum strain ATCC 12464 was used as control.

Three colonies were picked and suspended in saline solution to obtain a 0.5 McFarland turbidity standard. A sterile swab on an applicator stick was dipped into the standardized suspension of bacteria and then streaked in three directions over the entire surface of the $5-10 \%$ sheep blood agar to obtain a uniform inoculation. The antibiotic discs were placed onto the agar surface and no closer together than 24 $\mathrm{mm}$. The plates were incubated at $37^{\circ} \mathrm{C}$ for 24 hours in anaerobic conditions. After incubation, the diameters of the areas of inhibition were measured from the center of the discs. The Minimum Inhibitory Concentration 
(M.I.C.) was determined using commercially MIC Test Strips (Liofilchem Diagnostici) impregnated with a predefined concentration gradient of the same antibiotics used for antibiogram (0.002 - $256 \mu \mathrm{g} / \mathrm{mL})$. The strips were applied onto an inoculated agar surface, the preformed exponential gradient of an antimicrobial agent was immediately transferred to the agar matrix. After 18 -hour incubation or longer at $37^{\circ} \mathrm{C}$ in anaerobic conditions, a symmetrical inhibition ellipse centered along the strip was formed. The MIC was read directly from the scale in terms of $\mu \mathrm{g} / \mathrm{mL}$ at the point where the edge of the inhibition ellipse intersected the strip MIC Test Strip.

\section{RESULTS AND DISCUSSION}

$\mathrm{Cl}$. septicum is phenotypically very similar to $\mathrm{Cl}$. chauvoei. Besides, the two species have various antigens in common and share similar 16S rRNA gene sequences (99.3\% identity). It has been shown that the 16S-23S rDNA spacer region might be used to identify and differentiate pathogenic clostridia ( $\mathrm{Cl}$. chauvoei, $\mathrm{Cl}$. septicum, $\mathrm{Cl}$. novyi and $\mathrm{Cl}$. sordellii) in gas gangrene without restriction enzyme digestion of the PCR products [9]. The main difference between $\mathrm{Cl}$. septicum and $\mathrm{Cl}$. chauvoei is the presence of a 71-bp insert in the 16S-23S rDNA spacer region of $\mathrm{Cl}$. septicum [9]. We have therefore chosen this region to rapidly identify and differentiate pathogenic clostridia. The PCR amplification and then the sequencing of PCR products showed the presence of the 71-bp insertion and $100 \%$ nucleotide identity to the reference $\mathrm{Cl}$. septicum genes (GeneBank AB040716). The pathogen was therefore identified as $\mathrm{Cl}$. septicum.

Cl. septicum showed sensitivity to ampicillin, penicillin, cephalexin, erythromycin, marbofloxacin and rifampin, and intermediate sensitivity to amoxicillin, nalidixic acid, kanamycin, lincomycin, norfloxacin, and spiramycin. In contrast, it displayed resistance to colistin, gentamycin, tetracycline and sulfamethoxazole-trimethoprim with MIC $0.006-0.75$ (Liofilchem - CLSI M100-S24, 2014. Performance Standards for Antimicrobial Susceptibility Testing). These results are similar to those of a previous report [10]. This study also confirms the in vitro sensitivity to penicillin, which makes it the drug of choice, while cephalexin and rifampin may be used as alternative antimicrobials.

In the management of infection due to Cl. septicum, timely diagnosis and chirurgic curettage, associated with a broad-spectrum antibiotic therapy, were found to be sufficient for the survival and recovery of the infected animals. The antibiotic therapy and the practice of curettage implemented in the two surviving animals have been effective to resolve the infection. Therefore, the surgical resection of necrotic tissue proved to be important as it created an aerobic environment unfavorable for the survival of these anaerobic bacteria.

For the prevention of malignant edema, it is very important to prevent the entry of bacteria through lesions; great care should, therefore, be taken in situations in which the integrity of the skin or mucosa may be breached. In general, good quality straw bedding should be used, though in the postpartum area and the infirmary it is advisable to use other types of bedding, such as mattresses, which should be managed following strict hygiene principles. Moreover, it should be borne in mind that clostridia are more abundant in alluvial areas where the land is poorly drained and marshy (Hungerford 1990) and that the incidence of the disease is higher during periods of heavy rain.

\section{CONCLUSIONS}

This study reports four cases of postpartum malignant edema in Mediterranean buffaloes. The clinical signs and the results of culture and molecular analyses all confirmed the diagnosis of $\mathrm{Cl}$. septicum infection. The antibiotic therapy and the practice of curettage implemented in the two surviving animals were effective in resolving the infection. Antimicrobial treatments should be based on the results of susceptibility tests. In our case study, despite the use of sulfadiazine-trimethoprim to which $\mathrm{Cl}$. septicum was resistant, secondary bacterial complications were avoided. The surgical resection of necrotic tissue proved to be important as it created an aerobic environment unfavorable for the survival of these anaerobic bacteria.

\section{REFERENCES}

[1] Odani JS, Blanchard PC, Adaska JM, Moeller RB, Uzal FA Malignant edema in postpartum dairy cattle. $J$ Vet Diagn Invest 2009; 21: 920-4.

[2] Wayt LK. Parturient malignant edema in a cow. J Am Vet Med Assoc 1951; 119: 353-4.

[3] Hungerford TG. Clostridial Disease. In Hungerford's Diseases of Livestock. 9th Ed. McGraw-Hill Book Co. Sydney 1990; pp. 35-710.

[4] Tweten RK. Clostridium perfrigens beta toxin and Clostridium septicum alpha-toxin: their mechanism and possible role in pathogenesis. Vet Microbiol 2001; 82: 1-9. 
[5] Kennedy CL, Krejany EO, Young LF, O'Connor JR, Awad $\mathrm{MM}$, Boyd RL, et al. The a-toxin of Clostridium septicum is essential for virulence. Mol. Microbiol 2005; 57(5): 13571366.

[6] Hickey MH, Kwan RYQ, Awad MM, Kennedy CL, Young LF, Hall $\mathrm{P}$, et al. Molecular and cellular basis of microvascular perfusion deficits induced by Clostridium perfrigens and Clostridium septicum. PLoS Pathog 2008; 4(4): e1000045.

[7] Quinn PJ, Carter ME, Markey B, Carter GR. Antimicrobial Agents. In Clinical Veterinary Microbiology. Mosby-Year Book Europe Limited, London, 1994; pp. 95-208.
[8] Bauer AW, Kirby WM, Sherris JC, Turck M. Antibiotic susceptibility testing by a standardized single disk method. Am J Clin Pathol 1966; 45(4): 493-96.

[9] Sasaki Y, Yamamoto K, Kojima A, Norimatsu M, Tamura Y. Rapid identification and differentiation of pathogenic clostridia in gas gangrene by polymerase chain reaction based on the 16S-23S rDNA spacer region. Res Vet Sci 2000; 69: 289-94.

[10] Gabay EL, Rolfe RD, Finegold SM. Susceptibility of Clostridium septicum to 23 antimicrobial agents. Antimicrob. Agents Ch 1981; 20(6): 852-3.

DOI: https://doi.org/10.6000/1927-520X.2020.09.01

(C) 2020 D'Angelo et al.; Licensee Lifescience Global.

This is an open access article licensed under the terms of the Creative Commons Attribution Non-Commercial License (http://creativecommons.org/licenses/by-nc/3.0/) which permits unrestricted, non-commercial use, distribution and reproduction in any medium, provided the work is properly cited. 